\title{
Fab-7 functions as a chromatin domain boundary to ensure proper segment specification by the Drosophila bithorax complex
}

\author{
Kirsten Hagstrom, ${ }^{1}$ Martin Muller, ${ }^{2}$ and Paul Schedl \\ Department of Molecular Biology, Princeton University, Princeton, New Jersey 08544 USA
}

\begin{abstract}
Fab-7 deletions in the bithorax complex have a novel gain-of-function phenotype, typically transforming parasegment 11 (PS11) into PS12 identity. Genetic analysis indicates that removal of the Fab-7 element results in the fusion of the iab-6 (PS11) and iab-7 (PS12) cis-regulatory domains into a single regulatory domain that inappropriately regulates Abdominal-B in PS11. This has led to the hypothesis that Fab-7 is a chromatin domain boundary that normally functions to ensure the autonomous activity of the $i a b-6$ and $i a b-7$ cis-regulatory domains. We use several different enhancer blocking assays to demonstrate that Fab-7 has the insulating properties expected of a domain boundary. We define a minimal fragment of $F a b-7$ sufficient for enhancer blocking, and demonstrate that it is completely distinct from an adjacent Polycomb-dependent silencer. We compare $\mathrm{Fab}-7$ to the $s u(\mathrm{Hw})$ insulator element, and show that $\mathrm{Fab}-7$ enhancer blocking activity is intermediate between that of five and twelve reiterated binding sites for the $\mathrm{Su}(\mathrm{Hw})$ protein. These results support the model that Fab-7 functions as a domain boundary within the context of the bithorax complex, making Fab-7 one of the first boundary elements that is known to have an essential function in vivo.
\end{abstract}

[Key Words: Bithorax complex; Fab-7; chromatin domain boundary; insulator; suppressor of Hairy-wing]

Received September 19, 1996; revised version accepted November 1, 1996.

Body patterning in many organisms involves the development of segments whose unique identities are specified by homeotic genes. The precise expression patterns of the homeotic genes are crucial for generating a normal body plan, and the misregulation of these genes can result in dramatic transformations of one body segment into another. In Drosophila, the identity of parasegments in the posterior of the fly is controlled by the three homeotic genes of the bithorax complex (BX-C), Ultrabithorax $(U b x)$, abdominal-A (abd-A), and Abdominal- $B$ $(A b d-B \mid$ (Lewis 1978; Duncan 1987). The parasegmentspecific expression patterns of these three genes are generated by a complicated cis-regulatory region that spans a DNA segment of $300 \mathrm{~kb}$. This cis-regulatory region is organized in a series of nine parasegment-specific regulatory elements or domains, $a b x / b x, b x d / p b x, i a b-2$, $i a b-3, i a b-4, i a b-5, i a b-6, i a b-7$, and $i a b-8$ (Lewis 1978; Karch et al. 1985; Celniker et al. 1990; Sanchez-Herrero 1991). Each of these domains directs the expression of one of the three BX-C homeotic genes in a specific parasegment. For example, the $i a b-5, i a b-6$, and $i a b-7$ cisregulatory domains direct $A b d-B$ expression in parasegments (PS) 10, 11, and 12, respectively (Karch et al. 1985).

\footnotetext{
${ }^{1}$ Corresponding author.

${ }^{2}$ Present address: Department of Zoology and Animal Biology, University of Geneva, CH1211 Geneva, Switzerland.
}

As illustrated in Figure 1, these cis-regulatory domains are located downstream of the $A b d-B$ transcription unit, and as is the case for the other $\mathrm{BX}-\mathrm{C}$ cis-regulatory domains, their proximal-distal order along the chromosome corresponds to the anterior-posterior order of the parasegments they specify.

Bithorax complex gene regulation can be divided into two phases, initiation and maintenance. The choice of parasegment identity is made during the initiation phase, and depends upon the combinatorial action of the gap and pair-rule gene products found in each parasegment (Müller and Bienz 1992; Shimell et al. 1994; Casares and Sanchez-Herrero 1995). These gap and pairrule proteins sequentially activate the BX-C cis-regulatory domains in successively more posterior parasegments. For example, the set of regulatory proteins present in PS11 activates $i a b-6$ in this parasegment, but not $i a b-7$, whereas the regulatory proteins present in PS12 activate $i a b-7$. Because the products of the gap and pair-rule genes are present only transiently in the early embryo, the activity state selected during the initiation phase must be fixed by the programming of a maintenance system in each cis-regulatory domain. This maintenance system requires the Polycomb-Group $\left(P_{c}-G\right)$ and trithorax-Group (trX-G) genes (for review, see Paro 1990; Kennison 1993; Simon 1995). The products of the Pc-G genes function as negative regulators, maintaining the 


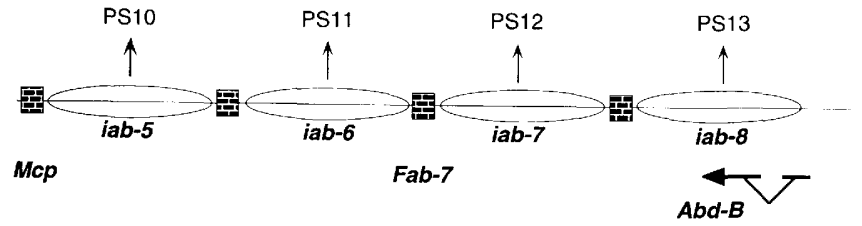

Figure 1. Schematic of BX-C cis-regulatory domains controlling $A b d-B$. The three genes of the BX-C are controlled by a large cis-regulatory region that is organized into a series of parasegment-specific domains. The cis-regulatory domains iab-5 to $i a b-8$ (ovals) control the $A b d-B$ gene (large arrow) to specify the identity of parasegments (PS) 10 through 13, respectively (Karch et al. 1985). Domain boundary elements (boxes) are thought to delimit the domains and allow their autonomous function by insulating the regulatory information in one domain from the influence of regulatory elements in the neighboring domain. The regions defined by the $M c p$ and $F a b-7$ mutations have been proposed to be such domain boundaries (Gyurkovics et al. 1990; Galloni et al. 1993; Vazquez et al. 1993).

inactive state of the homeotic genes, whereas the products of the $\operatorname{trx}^{-} G$ function as positive regulators, maintaining the active state. The $P c-G$ proteins appear to exert their regulatory effects by interacting with specific elements in each cis-regulatory domain, called polycomb response elements (PREs) (Simon et al. 1990; Busturia and Bienz 1993; Simon et al. 1993; Chan et al. 1994; Gindhart and Kaufman 1995). There may be equivalent or overlapping elements (TREs) for the trx-G proteins. Whether the programming of the maintenance system in each cis-regulatory domain involves the direct interaction of gap and pair-rule gene products with components of the PREs (and TREs) or requires some other intermediary factors is not known (Bienz 1992).

Mutations that inactivate one of the nine parasegment-specific regulatory domains transform the parasegment specified by that domain into a copy of the parasegment immediately anterior. Paralleling this transformation in segmental identity, the normal temporal and spatial pattern of the relevant $\mathrm{BX}-\mathrm{C}$ homeotic gene is replaced by the expression pattern found in the parasegment immediately anterior. For example, a mutation that deletes much of the $i a b-7$ cis-regulatory domain $\left(i a b-7^{S z}\right)$ transforms PS12 into a duplicated copy of PS11 (Galloni et al. 1993). As expected from this loss-of-function phenotype, the $A b d-B$ expression pattern normally seen in PS12 is replaced by a PS11 pattern, which is generated by the adjacent iab-6 cis-regulatory domain.

Whereas most mutations in the BX-C cis-regulatory domains cause a loss-of-function phenotype, the $\mathrm{Fab}-7$ alleles are exceptional in that they have a gain-of-function phenotype. Fab-7 was first defined by Fab-7 ${ }^{1}$, a $4-\mathrm{kb}$ deletion that removes sequences between the $i a b-6$ and iab-7 cis-regulatory domains (Gyurkovics et al. 1990; see Figs. 1 and 2). Subsequently, smaller deletions within the same region have been generated (Galloni et al. 1993; Karch et al. 1994). All of the Fab-7 alleles are dominant and lead to the transformation of PS11 into a duplicate copy of PS12. This phenotypic transformation arises from the inappropriate activation of the iab-7 cis-regu- latory domain in PS11 where iab-6 normally functions. As a consequence, $A b d-B$ is expressed in a PS12-pattern in PS11 (Galloni et al. 1993).

Why does a deletion that removes a region between cis-regulatory domains result in the ectopic expression of $A b d-B$ ? One possibility is that the sequences deleted by the Fab-7 mutations contain a parasegment-specific repressor that normally prevents the PS12-specific activators in $i a b-7$ from acting on $A b d-B$ in PS11 (Busturia and Bienz 1993; Zink and Paro 1995). However, the results of reversion analysis argue against this model. The Fab-7 phenotype can be reverted not only by second site mutations that inactivate $i a b-7$ but also by mutations that inactivate $i a b-6$ (Gyurkovics et al. 1990). These results indicate that both $i a b-6$ and $i a b-7$ contribute to the mutant phenotype and suggest that the deletion of the Fab-7 region causes inappropriate interactions between regulatory elements in the two domains. Thus an alternative hypothesis is that the Fab-7 region contains a chromatin domain boundary that functions to insulate the two domains /originally proposed in Gyurkovics et al. 1990|. In the absence of the Fab-7 boundary element, $i a b-6$ and $i a b-7$ no longer behave as autonomous units but instead fuse into a single cis-regulatory domain.

The idea that Fab-7 corresponds to a domain boundary is supported by studies on the Bluetail transposon /Galloni et al. 1993). This transposon contains a $U b x / l a c Z$ reporter and is inserted on the distal side of $\mathrm{Fab}-7$, just within the iab-7 cis-regulatory domain. Bluetail is expressed in an $A b d$ - $B$-like pattern in PS12, indicating that it is subject to control elements in the iab-7 cis-regulatory domain. However, because it is inactive anterior to PS12, the transposon apparently is insulated from control elements in the immediately adjacent iab-6 cis-regulatory domain (and also from elements in iab-5). Thus, like other elements that have been proposed to function as chromatin domain boundaries, Fab-7 appears to restrict enhancers from acting outside of their normal domain.

Although these findings suggest that sequences from the Fab-7 region act as a boundary within the context of the BX-C, it is important to establish whether these sequences can function as an autonomous boundary element in assays that are independent of other regulatory components from the $\mathrm{BX}-\mathrm{C}$. In the studies reported here, we use three different enhancer blocking assays to demonstrate that Fab-7 has boundary activity. We evaluate the strength of the Fab-7 boundary in different enhancerpromoter combinations, and in comparison with previously characterized boundaries. We also define a minimal Fab-7 fragment that can function as a boundary and show that it is distinct from an adjoining DNA segment that has silencer activity and appears to be the $i a b-7$ PRE.

\section{Results}

Fab-7 shows boundary function in an enhancer blocking assay

Whereas it is thought that the normal function of Fab-7 
Figure 2. Organization of the Fab-7 region and summary of enhancer blocking results obtained with white enhancer:mini-white transgenes. (A) The $F a b-7$ region of the $\mathrm{BX}-\mathrm{C}$ is depicted by the black bar between the PSIl regulatory domain $i a b-6$ at left, and the PS12 regulatory domain $i a b-7$ at right. This 3.35 HindIII-XbaI fragment includes one minor and three major nuclease hypersensitive sites (HS1, HS2, and HS3) whose limits are indicated by boxes (Galloni et al. 1993; Karch et al. 1994). Halfovals represent the presumed position of nucleosomes. The dotted lines above indicate the extent of three Fab-7 deletions: $F a b-7^{1}$ is the original deletion and transforms PS11 to PS12, whereas $F a b-7^{2}$ and $\mathrm{Fab}-7^{3}$ give a slightly less complete transformation (Gyurkovics et al. 1990; Galloni et al. 1993). The insertion site of the Bluetail transposon, which is subject to control by $i a b-7$ but not $i a b-6$, is shown /Galloni et al. 1993). Results presented here and elsewhere suggest that the two bracketed regions are the $F a b-7$ boundary and the $i a b-7$ PRE (this work; K. Hagstrom, M. Muller, and P. Schedl, in prep.). (H3) HindIII; (Nco) NcoI; (Pst) PstI; (Nsi) NsiI; (Apa) ApaI; (Aat) AatII; (Eae) EaeI; (Xba) XbaI. $(B-D)$ The basic P-element construct contains a white enhancer fragment that drives expression in the eye and testes (gray box), the miniwhite eye color gene (arrow), and scs' to minimize position effects from this side (open box) (Levis et al. 1985; Pirrotta 1988; Kellum and Schedl 1992). The extent of the Fab-7 fragment (black bar) in the test constructs is indicated by restriction sites that correspond to those in $A$. In all constructs the distance between the white enhancer and mini-white depends on the size of the inserted fragment (thin line does not indicate preserved spacing|. At right are shown the number of independent transgenic lines with an eye color lighter than that of the basic $w \# 2$ control over the total number of lines generated for each construct.

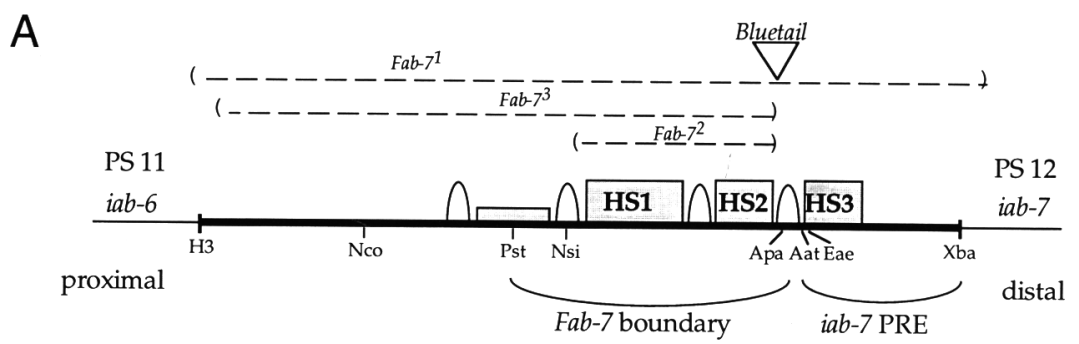

B

Enhancer blocking (\#light eyes/total)

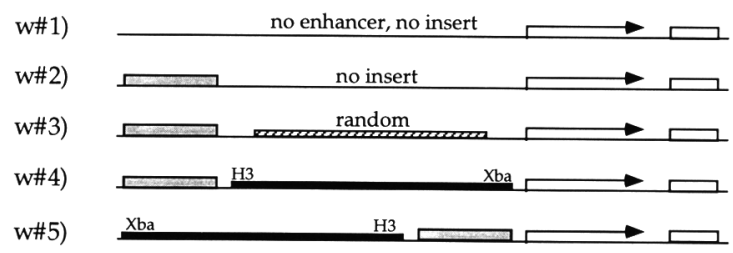

$-\quad(5 / 5)$

$-(0 / 6)$

$-\quad(0 / 5)$

$+(12 / 24)$

$-\quad(1 / 11)$

C

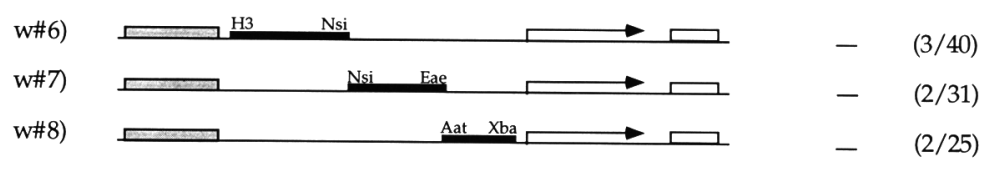

D

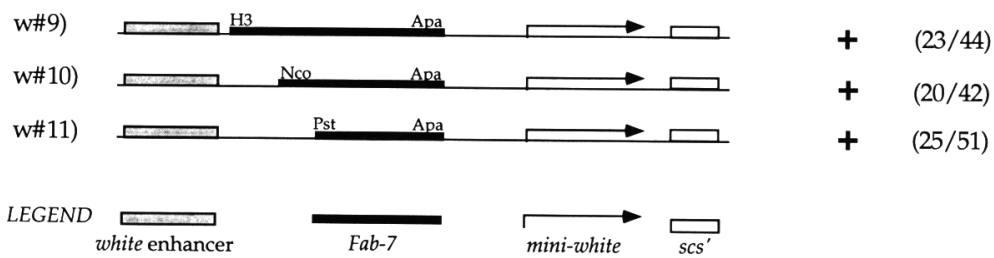

is to insulate the $i a b-6$ and $i a b-7$ domains, studies on the Bluetail transposon indicate that $\mathrm{Fab}-7$ can also prevent enhancer-promoter interactions (Galloni et al. 1993). Thus it seemed appropriate to use an enhancer-blocking assay (Kellum and Schedl 1992). This assay tests whether a putative boundary element is able to impede enhancerpromoter communication when interposed between an enhancer and a reporter gene. DNA fragments containing a boundary or insulator element should block enhancer-promoter interactions, and reduce reporter-gene expression. If the fragment does not contain a boundary, expression should be equivalent to that observed when only the enhancer and reporter are present. A boundary or insulator element can be distinguished from an ele- ment that acts as a silencer by its position dependence. A boundary element can only block enhancer-promoter interactions when placed between the enhancer and promoter; when placed upstream of the enhancer, it should have no effect on enhancer-promoter interactions. In contrast, a silencer is expected to attenuate enhancer and/or promoter activity even from an upstream position.

For the enhancer blocking assay we used a mini-white reporter gene and an enhancer from the white locus that drives expression in the eye and testes (Levis et al. 1985; Pirrotta 1988). In the first series of constructs (Fig. 2B) we tested a fragment of Fab-7 that spans most of the $4-\mathrm{kb}$ region deleted in the original $F a b-7^{1}$ mutation. Previous 
chromatin mapping studies identified a set of three strong and several weak nuclease hypersensitive sites within the region deleted in Fab $-7^{1}$ (Galloni et al. 1993). Two of the strong and one of the more prominent weak hypersensitive sites are located centromere proximal to the Bluetail transposon, whereas the third strong hypersensitive site is distal to the transposon (see Fig. 2A). Because we suspected that the Fab-7 boundary might be associated with this unusual chromatin structure, we selected a 3.3-kb HindIII-XbaI fragment that includes all of these nuclease hypersensitive sites for our initial experiments. The experimental construct in the series (w\#4) contains this 3.3-kb Fab-7 fragment between the white enhancer and the mini-white reporter. Control constructs $\mathrm{w} \# 1$ and $\mathrm{w} \# 2$ allowed us to determine the level of mini-white expression with or without the enhancer. Construct $w \# 3$ controls for the possible effects of distance by inserting a random 2.6-kb DNA fragment between the enhancer and reporter. Finally, control construct $\mathrm{w} \# 5$ distinguishes between boundary and silencer activity by placing the 3.3 -kb Fab-7 fragment upstream of the enhancer.

Figure 3 shows the eye color phenotype of a representative transgenic line for each construct. As expected from previous studies on mini-white (Kellum and Schedl 1991), transgenic lines that carry the mini-white gene but no enhancer have a light yellow eye color (Fig. 3, $\mathrm{w \#}$ 1). When the white enhancer is included in the construct, the expression of mini-white is stimulated and the eye color is orange/red in all 5 of the lines (Fig. 3, w\#2; Fig. 2B). The same orange/red eye color is observed when a random DNA fragment is inserted between the white enhancer and the mini-white gene (Fig. 3, w\#3; Fig. 2B). Hence, it would appear that a modest increase in distance has no effect on enhancer-promoter interaction in this reporter system.

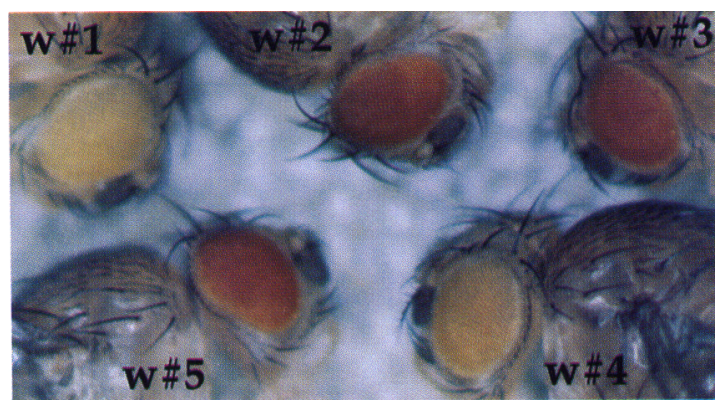

Figure 3. Eye colors of representative transgenic lines containing white enhancer:mini-white constructs $\mathrm{w} \# 1-5$. Eye colors of heterozygous 1-day-old females are shown. (w\#1) With no white enhancer, eyes have the yellow color characteristic of basal mini-white expression. (w\#2) The white enhancer elevates mini-white expression to give an orange/red color. (w\#3) An insert of random DNA between the enhancer and promoter has no effect and gives the same orange/red eye color as $w \# 2$. (w\#4) The entire HindIII-XbaI fragment of Fab-7 between the enhancer and promoter reduces eye color to nearly the same yellow as with no enhancer. (w\#5) Fab-7 cloned upstream of the enhancer gives an orange/red eye color like the control.
A different result was obtained when the Fab-7 DNA fragment was inserted between the enhancer and promoter (w\#4). For this construct, 12 of the 24 transgenic lines have reduced mini-white expression and have an eye color lighter than the control constructs $w \# 2$ and $\mathrm{w} \# 3$ that contain the white enhancer (Fig. 3). Similar effects on white expression were observed in the testes (not shown). A reduction in white expression in the eye and testes is only found when the Fab-7 fragment is placed between the enhancer and promoter. When the Fab-7 fragment is placed upstream of the white enhancer $(\mathrm{w} \# 5)$, the eye color phenotype in most of the transgenic lines $(10 / 11)$ was the same as that observed in the construct with just the white enhancer (Fig. 2B; Fig. 3).

Taken together these findings indicate that the $3.3-\mathrm{kb}$ Fab-7 fragment is able to interfere with enhancer-promoter interactions when interposed between the white enhancer and the mini-white reporter but has no effect on mini-white expression when placed upstream of the white enhancer. There were, however, two troubling observations.

The first is the observation that only half of the construct $\mathrm{w} \# 4$ lines had the eye and testes pigmentation phenotype expected for an insulator element. At least two factors could account for this result. One would be position effects arising from regulatory elements located downstream of the mini-white transgene (c.f. Roseman et al. 1993). Although scs' is located $3^{\prime}$ of mini-white in our vector, this element is not able to insulate completely against chromosomal position effect (Vazquez and Schedl 1994). The other factor may be the relative strength of the Fab-7 element. Incomplete enhancer blocking activity has been observed in the white assay system for boundary elements whose activity has been partially compromised by deletion (Vazquez and Schedl 1994). This issue is considered further below.

The second troubling observation came when we compared the eye color of animals heterozygous and homozygous for the Fab-7 transgenes w\#4 and w\#5. Normally the eye color of animals homozygous for a miniwhite transgene are darker than the eye color of heterozygous animals. However, in about $10 \%$ of the lines the eye color is lighter, not darker, in homozygous animals. Moreover, in some cases, regions of the eye are completely white indicating that even the basal activity of the mini-white promoter has been shut off. This phenomenon is called pairing-sensitive repression, and has been observed when PREs are included in mini-white constructs (Kassis et al. 1991; Fauvarque and Dura 1993; Chan et al. 1994; Gindhart and Kaufman 1995). Consistent with the presence of a PRE in the 3.3-kb HindIII$X b a I$ fragment, we found that the pairing-sensitive repression of mini-white requires $P c-G$ genes (K. Hagstrom, M. Muller, and P. Schedl, in prep.). Other workers have also detected a PRE-like silencer in constructs containing large fragments from the $F a b-7$ region of the BX-C (Busturia and Bienz 1993; Zink and Paro 1995). Although this PRE silencing activity would not seem to account for the fact that Fab-7 fragments block the action of the white enhancer only when interposed be- 
tween the enhancer and the mini-white reporter, it would nevertheless complicate interpretation of our experiments.

Silencing activity maps to the distal side of the Bluetail transposon

One way to resolve the question of whether Fab-7 is a boundary or a silencer is to determine whether these two activities can be separated. For this purpose we divided the 3.3-kb Fab-7 fragment into three smaller fragments and introduced them into the mini-white reporter vector (Fig. 2C, also see K. Hagstrom, M. Muller, and P. Schedl, in prep.). The first of these $(w \# 6)$ extends from the HindIII site at the proximal end of the $3.3-\mathrm{kb}$ fragment to just beyond the minor nuclease hypersensitive site; the second (w\#7) spans the first two major proximal nuclease hypersensitive sites, and the third $(w \# 8)$ contains the third major nuclease hypersensitive site just beyond the site of insertion of the Bluetail transposon. Of these three subfragments only $w \# 8$ shows pairing sensitive silencing in our mini-white reporter system. About a third of the w\#8 lines had reduced mini-white expression when the transgene is homozygous, and, like the 3.3-kb HindIII-XbaI fragment, this silencing depends upon $P C-G$ proteins. In studies described in detail elsewhere we have shown that fragment $w \# 8$ most likely contains the PRE for the iab-7 cis-regulatory domain (K. Hagstrom, M. Muller, and P. Schedl, in prep.). It should be noted that, whereas fragment $w \# 8$ has silencing activity, it does not have enhancer-blocking activity (Fig. $2 \mathrm{Cl}$. Although fragments $\mathrm{w} \# 6$ and $\mathrm{w} \# 7$ had no detectable silencing activity, they also showed no evidence of boundary function (Fig. 2C).

Fab-7 boundary activity maps to the hypersensitive sites on the proximal side of the Bluetail transposon

In previous studies two other Fab- 7 alleles, $F a b-7^{2}$ and $F a b-7^{3}$, were generated by imprecise excision of the Bluetail transposon (Galloni et al. 1993). Both of these deletions extend proximally from the Bluetail transposon, and remove the major hypersensitive sites HSI and HS2, but leave HS3 (and the iab-7 PRE) intact (Fig. 2A). The DNA sequence removed in the smaller deletion, Fab $-7^{2}$, corresponds closely to the Nsil-Eael fragment contained in construct $w \# 7$. Consequently, we had anticipated that this fragment would have enhancer blocking activity; however, as described in the previous section it did not. This suggests that the sequences removed by $\mathrm{Fab}-7^{2}$ may be essential, but not sufficient, for boundary activity. If this hypothesis is correct, then we might be able to reconstitute boundary activity by including additional proximal sequences.

For this purpose we tested a fragment which extends from HindIII to ApaI, and covers the DNA sequences removed in the slightly larger $F a b-7^{3}$ deletion (w\#9). We found that this HindIII-ApaI fragment has enhancerblocking activity (see Fig. 2D), and, as was observed for the $3.3 \mathrm{Fab}-7$ fragment (w\#4), $\sim 50 \%$ of the transgenic lines had reduced eye pigmentation. However, unlike the 3.3-kb fragment, the HindIII-ApaI fragment does not show pairing-sensitive repression of mini-white. This finding confirms the localization of the PRE to distal side of the Bluetail transposon and demonstrates that boundary and silencing activities are distinct.

To delimit further the DNA sequences that are sufficient to confer boundary activity, we generated two additional constructs (Fig. 2D). Contruct w\#10 has a fragment that includes the major hypersensitive sites HSI and HS2, plus the minor hypersensitive site. The PstIApaI fragment in w\#11 includes all of HS1 and HS2, but only about half of the minor hypersensitive site. As indicated in Fig. 2D, enhancer blocking activity roughly equivalent to that of the entire $3.3-\mathrm{kb}$ fragment was observed for both of these smaller Fab-7 fragments. Moreover, neither of these fragments showed any evidence of silencer activity. Thus, the PstI-ApaI fragment in construct $\mathrm{w} \# 1 \mathrm{l}$ defines the maximum limits of the Fab-7 clement sufficient for enhancer blocking in this assay.

Fab-7 functions as a boundary in a ftz:hsp70/lacZ enhancer blocking assay

Although the results described in the previous sections indicate that the Fab-7 element can block enhancer-promoter communication, it could be argued that our miniwhite system measures boundary activity at stages of development and/or in cell types that are not especially relevant to $\mathrm{BX}-\mathrm{C}$ regulation. Consequently, it was important to determine whether Fab-7 also has enhancerblocking activity at developmental stages and in cell types in which the element would normally be required. For this purpose, we chose the fushi tarazu (ftz):hsp70/ lacZ reporter diagrammed in Fig. 4A. This reporter has two enhancers from $f t z$, the upstream element (UPS) that activates the $h s p 70$ promoter in even-numbered parasegments of germband-extended embryos, and the neurogenic element (NE) that activates the $h s p 70$ promoter in the central nervous system (CNS) of germband-retracted embryos (Hiromi et al. 1985; Hiromi and Gehring 1987). The ftz:hsp70/lacZ reporter is also advantageous because it can be used to ask whether the activity of the Fab-7 boundary is modulated along the anterior-posterior axis (c.f. Peifer et al. 1987).

We generated four constructs with the $f t z$ :hsp $70 / 1 a c Z$ vector (Fig. 4A). The first, fh\#1, controls for distance effects in this assay system and has a random DNA fragment inserted between the $f t z$ enhancers and the $h s p 70$ promoter. The second, fh\#2, tests the enhancer-blocking function of Fab-7. It has a Fab-7 fragment (Ncol-ApaI) that includes the boundary but not the silencer. Finally, in constructs fh $\# 3$ and fh $\# 4$ we have inserted a constitutively active insulator, the suppressor of Hairy-wing $(s u(H w))$ element from the gypsy transposon, between the $f t z$ enhancers and the $h s p 70$ promoter (Roseman et al. 1993; Scott and Geyer 1995). Besides providing a control for any parasegmental regulation of $\mathrm{Fab}-7$ boundary activity, these two constructs were designed to address an issue raised by the mini-white assay, namely the strength of Fab-7 enhancer blocking activity. It is thought that the blocking activity of the su(Hw) element 
A

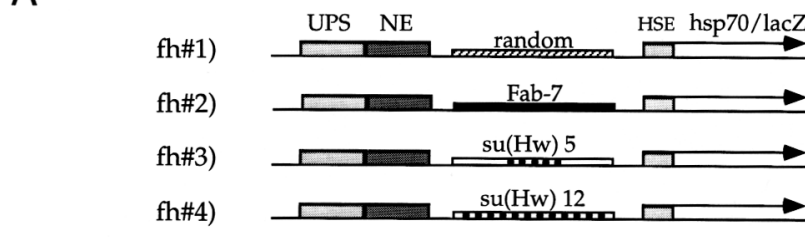

B

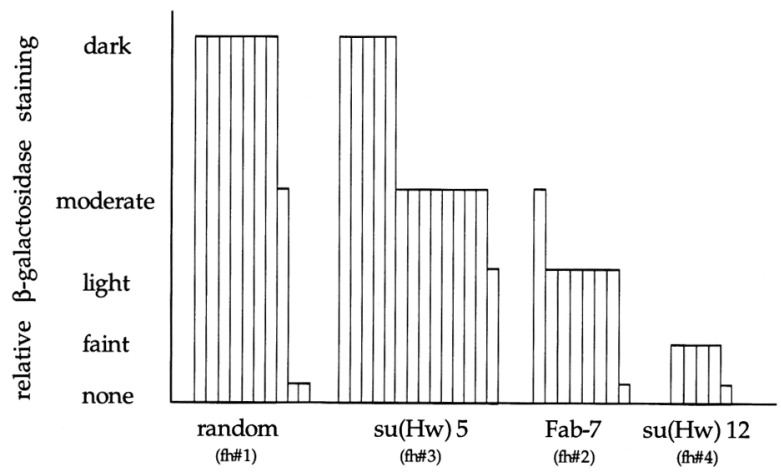

Figure 4. $f t z$ enhancer:hsp $70 /$ lac $Z$ reporter construct design and enhancer-blocking results. (A) The basic ftz:hsp70/lacZ P-element construct contains two enhancers from the $f t z$ gene. The UPS (lightly shaded box) drives expression in seven stripes in germ band-extended embryos and the NE (darkly shaded box) drives expression in the CNS of germband-retracted embryos (Hiromi et al. 1985; Hiromi and Gehring 1987). The reporter is an $h s p 70(-194)$ promoter fragment, which includes the HSE upstream sequences required for heat shock induction, fused to the lac $Z$ gene. The fragments tested between the enhancers and promoter are as follows: (fh\#1) 1.4-kb random DNA control fragment from Bic-D (Suter et al. 1989); (fh\#2) 1.7-kb Fab-7 ApaI-NcoI boundary fragment; (fh\#3) 390-bp fragment from gypsy (and pUC18) with only $5 \mathrm{su}(\mathrm{Hw}$ ) binding sites; (fh\#4) 440-bp fragment from gypsy with all $12 \mathrm{su}(\mathrm{Hw})$ binding sites (Spana et al. 1988). (B) The relative levels of $\beta$-galactosidase staining for each of the fh constructs were scored as none, faint, light, moderate, and dark. All lines were scored simultaneously and compared relative to selected control lines. Each individual transgenic line is shown as one bar above the name of the construct, and the relative level of staining of each line is indicated by the height of the bar. All lines that gave no staining were treated with heat shock, and only the su(Hw)12 line could be induced, suggesting that only in this line is the lack of staining attributable to strong enhancer blocking.

depends upon the number reiterated binding sites for the $\mathrm{Su}(\mathrm{Hw})$ protein (Peifer and Bender 1988; Smith and Corces 1992). Construct fh\#3 has five $\mathrm{Su}(\mathrm{Hw})$ proteinbinding sites, whereas construct fh\#4 has twelve binding sites, the number of sites typically found in gypsy transposons. The pattern of $\beta$-galactosidase expression in transgenic embryos from a line representative of each of the four $f t z$ :hsp70/lacZ constructs is shown in Figure 5.

For the random DNA control, fh $\# 1$, we observe seven darkly stained $\beta$-galactosidase stripes in germband-extended embryos. The level of $\beta$-galactosidase expression in these embryos is indistinguishable from that observed in embryos transgenic for the starting ftz:hsp $70 / 1 a c Z$ vector (not shown). This would suggest that the activation of the hsp 70 promoter by the ftz UPS enhancer is not significantly perturbed by a small increase in distance. This also appears to be true for the $f t z$ NE enhancer element. This enhancer drives high levels of $\beta$-galactosidase expression in the CNS of germband-retracted $\mathrm{fh} \# \mathbf{1}$ embryos. As indicated in the graphs in Figure $4 \mathrm{~B}$, seven of the ten fh\#1 transgenic lines had high levels of stripe and CNS $\beta$-galactosidase expression like that observed in the representative embryos shown in Figure 5 (dark in Fig. 4B). For the three remaining lines, one had an intermediate level of $\beta$-galactosidase expression (moderate, like that observed for fh\#3 embryos in Fig. 5), whereas no expression could be detected in the other two lines (none). The lack of expression in these two lines is likely to be attributable to some type of position effect (see below).

The level of $\beta$-galactosidase expression is reduced relative to the control in embryos transgenic for the construct containing five binding sites for the $\mathrm{Su}(\mathrm{Hw})$ protein, fh\#3. As can be seen for one of the fh\#3 transgenic lines in Figure 5 , the intensity of $\beta$-galactosidase staining in the seven stripes and also in the CNS is decreased compared to that observed for the transgenic line carrying the random DNA control, fh\#1. The results for all of the fh\#3 transgenic lines are summarized in Figure 4B. The level of stripe and CNS $\beta$-galactosidase expression in 8 of the 14 lines resembles the fh\#3 embryos shown in Figure 5, and these fall into the moderate class. Five lines had levels of expression equivalent to the fh\#1 control and these are in the dark class, whereas the one remaining line had only a low level of expression and is in the light class. Thus a $s u(H w) 5$ mer has a weak enhancer-blocking activity.

Much stronger enhancer blocking is observed for the construct containing twelve $s u(H W)$ binding sites, fh\#4. As illustrated in Figure 5, the $s u(H w) 12$ mer causes a substantial decrease in the level of $\beta$-galactosidase expression. The characteristic stripe and CNS staining patterns generated by the UPS and NE enhancers are mostly eliminated, and only some residual staining is evident. Four of the five fh\#4 lines have stripe and CNS expression patterns like those shown in Figure 5 (faint), whereas $\beta$-galactosidase expression is reduced even further in the fifth line. These findings demonstrate that the enhancer-blocking activity of the $s u(H w)$ element depends upon the number of $s u(H w)$ binding sites.

The level of $\beta$-galactosidase expression in embryos transgenic for the Fab-7 construct, fh\#2, is in between that observed for the constructs containing five and twelve su( $H w)$ binding sites. As shown in Figure 5, the Fab-7 boundary substantially reduces both stripe and CNS staining. Although the level of stripe or CNS expression is not precisely the same in all parasegments of fh\#3 embryos, equivalent parasegmental-specific differences are observed for the control constructs containing the constitutively active $s u(\mathrm{Hw})$ boundary or random DNA (Fig. 5). Thus, these parasegmental differences most likely reflect some intrinsic property of the $f t z$ UPS and NE enhancer elements, rather than a parasegmental 
Figure 5. $\beta$-galactosidase staining of representative lines transgenic for $f t z: h s p 70 / l a c Z$ constructs fh\#1-4. The UPS enhancer-driven seven-stripe staining pattern in germband-extended embryos is shown at left, and the NE enhancer-driven CNS staining pattern in germband-retracted embryos is shown at right. Embryos were stained simultaneously, and a representative of the average level of staining for each th construct is shown. Anterior is at left, and posterior at right.
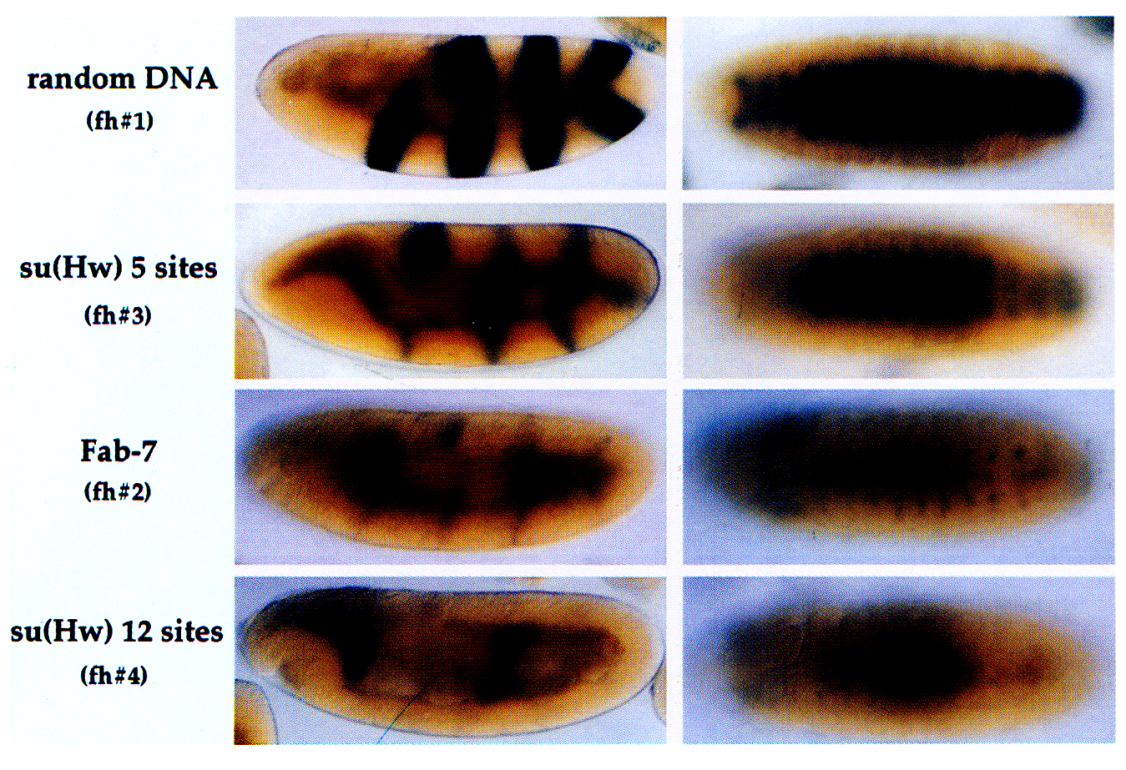

specificity of $\mathrm{Fab}-7$. As indicated in Figure $4 \mathrm{~B}, \beta$-galactosidase expression in six of the eight Fab-7 transgenic lines resembles the line shown in Figure 5 (light). Of the two remaining lines, one showed a moderate level of $\beta$-galactosidase expression much like that observed for constructs containing five $\mathrm{Su}(\mathrm{Hw})$ protein binding sites (see Fig. 5), whereas no $\beta$-galactosidase expression could be detected in the other line (see below). These results demonstrate that $\mathrm{Fab}-7$ can block enhancer-promoter interactions in a second independent assay system, and that the relative enhancer-blocking activity of the $\mathrm{Fab}-7$ boundary is in between that generated by five and twelve copies of the $\mathrm{Su}(\mathrm{Hw})$ protein binding site.

\section{The Fab-7 boundary does not repress intrinsic promoter or enhancer activity}

One criterion that distinguishes a boundary or insulator element from a silencer is whether it has any effect on the intrinsic activity of the promoter or the enhancer. A boundary element is expected to reduce reporter gene expression by impeding enhancer-promoter communication. In contrast, silencers exert their effects on reporter gene expression either by repressing the promoter, or by inactivating the enhancer. The $h s p 70$ promoter fragment used in our $f t z$ reporter construct contains not only the sequences required for basal promoter activity (the GAGA binding sites, the TATA box, and the transcription start site) but also the upstream HSE elements that activate the promoter in response to heat shock ( $\mathrm{Si}$ mon et al. 1985). Hence we can use heat induction to test whether the Fab-7 element has any inhibitory effect on the activity of either the basal hsp 70 promoter or the HSE elements.

As illustrated in Figure 6, the $h s p 70$ promoter in the construct containing the $\mathrm{Fab}-7$ boundary is efficiently induced by heat shock. The level of induction in the
Fab-7 (fh\#2) transgenic embryos appears to be equivalent to that observed in embryos transgenic for either the random DNA control (fh\#l) or the $s u(H w)$ fragment (fh\#4). This is true for both germ band-extended embryos (Fig. 6), in which Fab-7 blocks hsp 70 promoter activation by the ftz UPS enhancer, and for germ bandretracted embryos (not shown), in which Fab-7 blocks $h s p 70$ promoter activation by the $f t z \mathrm{NE}$ enhancer. Because the $h s p 70$ promoter can be induced efficiently, the enhancer-blocking activity of Fab-7 in the $f t z$ assay cannot be attributed to the silencing of the basal $h s p 70$ promoter. Similarly, the fact that the HSE elements in the fh\#2 constructs appear to be fully active would suggest that the Fab-7 fragment is unlikely to function by directly inhibiting enhancers.

We also tested whether the $h s p 70$ promoter can be activated by heat shock in the three exceptional transgenic lines (two control fh\#1 lines, and one Fab-7 fh\#2 line) that showed little stripe and CNS $\beta$-galactosidase expression. We found that $\beta$-galactosidase could not be induced by heat shock in either of these control lines (fh\#1) or in the Fab-7 (fh\#2) line (not shown). This would suggest that the basal promoter of these particular transgenes is inactivated by some type of position effect.

\section{Blocking activity depends upon the} enhancer-promoter combination

The $f t z$ reporter used in the blocking assays described above combines the $f t z$ enhancers with a heterologous $h s p 70$ promoter. We wondered whether the Fab-7 and $s u(H w)$ boundaries would have an equivalent blocking activity when assayed in a construct that instead combines the $f t z$ enhancers with the homologous $f t z$ promoter (ff\#1). As shown in Figure 7A, we placed the Fab-7 $(\mathrm{ff} \# 2)$ and the $s u(H w) 12$ mer $(\mathrm{ff} \# 3$ ) boundaries between the NE and UPS enhancers and the $f t z$ promoter in this $f t z$ ftz/lacZ reporter. This reporter differs from the 


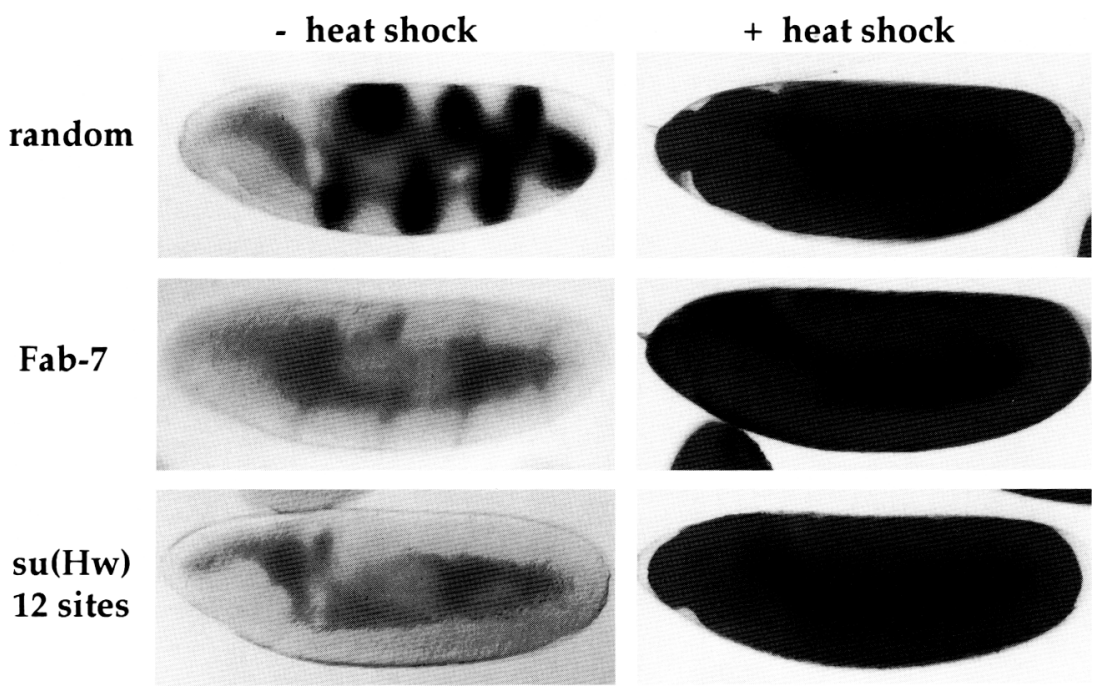

Figure 6. The Fab-7 boundary does not inactivate the $h s p 70$ promoter or the HSE elements. Embryos transgenic for ftz:hsp 70/ $1 a c Z$ constructs containing random DNA, the Fab-7 ApaI-NcoI fragment, and the su(Hw) 12 binding site fragments are shown with (right) and without (left) heat shock at $37^{\circ}$ for $1 \mathrm{hr}$. In all constructs heat shock treatment induces equivalent high levels of $\beta$-galactosidase expression. $f t z: h s p 70 / 1 a c Z$ reporter in that it also contains the $f t z$ Zebra element that is located immediately adjacent to the $f t z$ basal promoter (Hiromi et al. 1985; Hiromi and Gehring 1987). In pre-germ band-extended embryos, the Zebra element directs $\beta$-galactosidase expression in seven stripes, and this makes it difficult to determine whether a boundary element is able to block the stripe expression generated later in development by the UPS enhancer. For this reason, we only examined the effects of the boundary elements on the NE-dependent CNS expression.

The results obtained with the $f t z: f z / l a c Z$ reporter are somewhat different from the $f t z: h s p 70 / l a c Z$ reporter (Fig. 7B). First, the level of $\beta$-galactosidase activity observed in the CNS of embryos transgenic for the starting ftz:ftz/lacZ reporter appeared to be somewhat less than that found in embryos transgenic for the ftz:hsp70lacZ reporter. Second, although the Fab-7 boundary is able to
A

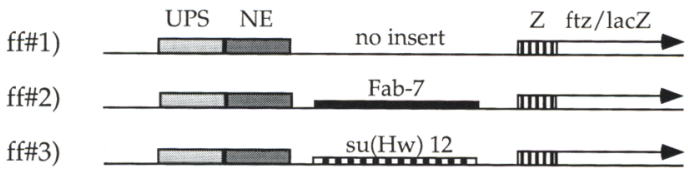

B

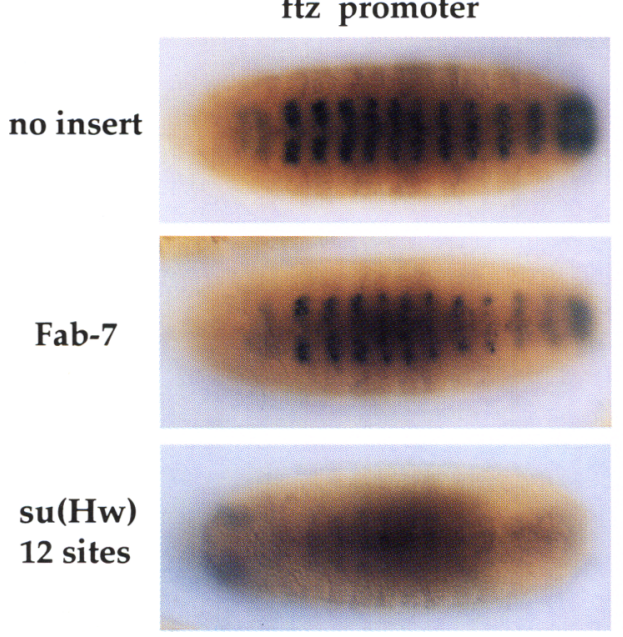

hsp 70 promoter

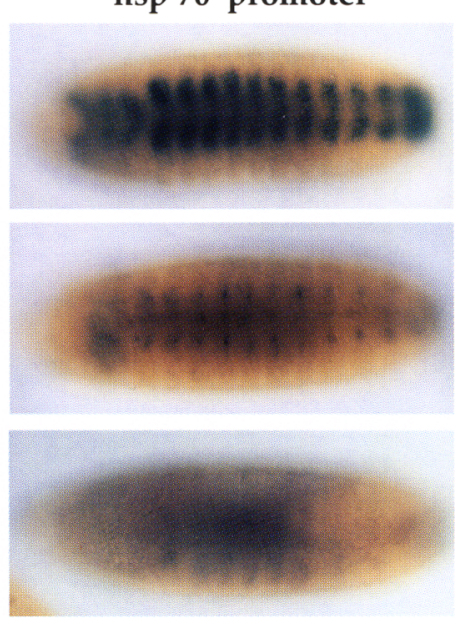

Figure 7. ftz:ftz promoter/lacZ construct design and comparison of enhancer blocking to ftz:hsp70 promoter/lacZ constructs. (A) The basic $f t z: f t z / l a c Z$ reporter construct contains the same UPS and NE enhancers as in the $f t z: h s p 70 / l a c Z$ construct /shaded boxes; also see Fig. 4). The promoter fused to lacZ (arrow), however, is derived from the $f t z$ upstream region and contains basal promoter elements as well as the zebra element enhancer (striped box) that drives a seven-stripe pattern in early embryos (Hiromi et al. 1985; Hiromi and Gehring 1987). Because of this additional stripe enhancer, we only assayed blocking of the NE enhancer. $(B)$ The $\beta$-galactosidase pattern generated by the NE is shown in germband-extended embryos transgenic for $f t z$ promoter/ lacZ constructs (left) or hsp 70 promoter/lacZ constructs (right). Without inserts (top), the basic $f t z$ promoter construct has slightly weaker staining than the basic $h s p 70$ promoter construct. The insertion of the ApaI-NcoI Fab-7 fragment (middle) causes a slight reduction of $N E$ driven staining in the $f t z$ promoter construct, and a greater reduction in the hsp 70 promoter construct. The insertion of 12 su(Hw) binding sites (bottom) causes nearly complete loss of staining in both, although this reduction is slightly more pronounced in the hsp 70 promoter construct. 
block NE-ftz promoter interactions, the boundary does not appear to function as effectively in the $f t z$ promoter construct as it does when the NE enhancer is combined with the heterologous $h s p 70$ promoter. This can be seen by comparing CNS expression in the control and experimental embryos in Figure 7B. Third, the $s u(H w)$ insulator also appears to be slightly less effective in the $f t z: f t z / l a c Z$ reporter (Fig. 7B), although the difference in $s u(H w)$ activity in the $h s p 70$ and $f t z$ promoter constructs is less pronounced than it is for Fab-7.

\section{Proteins that influence su(Hw) enhancer blocking do not affect Fab-7 blocking}

Two trans-acting factors have been implicated in the blocking activity of the su(Hw) element. One of these is encoded by the $s u(H w)$ locus, and loss-of-function mutations in this gene reduce or eliminate blocking (Modolell et al. 1983; Roseman et al. 1993). The other is encoded by the mod(mdg4) locus. Mutations in mod$(m d g 4)$ have been reported to have an unusual effect on the enhancer-blocking activity of a gypsy transposon inserted in the yellow locus (the $y^{2}$ allele) (Gerasimova et al. 1995). In the mod(mdg4) background the $s u(H w)$ element within the gypsy transposon mimics a bidirectional silencer and interferes with the activity of yellow enhancers located both upstream and downstream of the transposon insertion.

We used the $f t z: h s p 70 / 1 a c Z$ reporter to test whether either of these genes is also important for Fab-7 boundary function. As expected, we found that mutations in $s u(H w)$ relieve the blocking activity of reiterated $s u(H w)$ binding sites (compare wt with $\mathrm{su}(\mathrm{Hw})^{-}$in Fig. 8). For example, the 12 mer largely eliminates $\beta$-galactosidase expression in a wild-type background; however, in a $s u(H w)$ mutant background, stripe expression is restored to a level equivalent to that of a $f t z: h s p 70 / 1 a c Z$ construct containing random DNA /not shown; note that levels of expression appear lower than in other figures because embryos in Fig. 8 are heterozygous for the transgene). Because studies on $y^{2}$ suggest that the $\bmod (\operatorname{mdg} 4)$ mutation causes the gypsy element to mimic a silencer, we expected that the mutation might silence the $\mathrm{ftz}$ enhancers in reporters containing the reiterated $s u(H w)$ binding sites, accentuating the apparent blocking activity (Gerasimova et al. 1995). However, we obtained the opposite result. Mutations in $\bmod (\operatorname{mdg} 4)$ restore $\beta$-galactosidase expression in the $s u(H w)$ lines (Fig. 8). This finding suggests that the enhancer-blocking activity of the reiterated $s u(H w)$ binding sites in the $f t z: h s p 70 / l a c Z$ reporter requires the $\bmod (\operatorname{mdg} 4)$ protein. Unlike the $s u(H W)$ binding sites, the enhancer blocking activity of $F a b-7$ is not noticeably affected by mutations in either $\mathrm{su}(\mathrm{Hw})$ or $\bmod (\operatorname{mdg} 4)$ (Fig. 8). This suggests that a different set of proteins are required for the boundary function of $s u(H w)$ and Fab-7.

\section{Discussion}

\section{The Fab-7 mutation disrupts a domain boundary}

A growing body of evidence indicates that eukaryotic chromosomes are subdivided into structurally and functionally distinct domains. In addition to providing a mechanism for organizing and compacting the large eukaryotic chromosomes inside the nucleus, the domain organization of the chromatin fiber can have a significant impact on gene regulation. For example, the parasegment-specific expression of the homeotic genes in the bithorax complex of Drosophila appears to depend upon the subdivision of the complex into a series of functionally independent cis-regulatory domains. This subdivision into autonomous domains is illustrated by the expression patterns of enhancer trap transposons integrated in different domains of the complex (Galloni et al. 1993; McCall et al. 1994). These enhancer traps are subject to regulatory elements located within the same domain, but are insensitive to regulatory elements in adjacent domains.

What ensures the autonomy of each domain? One mechanism may be the insulation of the domains by elements that can function as chromatin boundaries. Previous genetic studies have suggested that the Fab-7
Figure 8. Mutations in the su(Hw) and $\bmod (m d g 4)$ proteins relieve enhancer blocking by $s u(\mathrm{Hw})$ binding sites, but have no effect on $\mathrm{Fab}-7$ blocking activity. $\beta$-galactosidase staining patterns of germband-extended embryos heterozygous for $\mathrm{ftz}$ :hsp 70/lac $Z$ constructs containing $12 \mathrm{su}(\mathrm{HW})$ binding sites (fh\#4, top rowl, five $s u(H w)$ binding sites (fh\#3, middle row), and the Fab-7 boundary (fh\#2, bottom row). In a wildtype background ( $w \mathrm{t}$, left column), these fragments show various degrees of staining attributable to enhancer blocking (as shown for homozygous embryos in Fig. 5). In a su(Hw) loss-of-function mutant background (su(Hw) ${ }^{-}$, middle column), and in a mod(mdg4) mutant background (mod ${ }^{-}$, right column) $\beta$-galactosidase levels increase in constructs containing $s u(H w)$ fragments. Neither mutation affects staining of embryos containing Fab-7 (bottom row, last two columns).

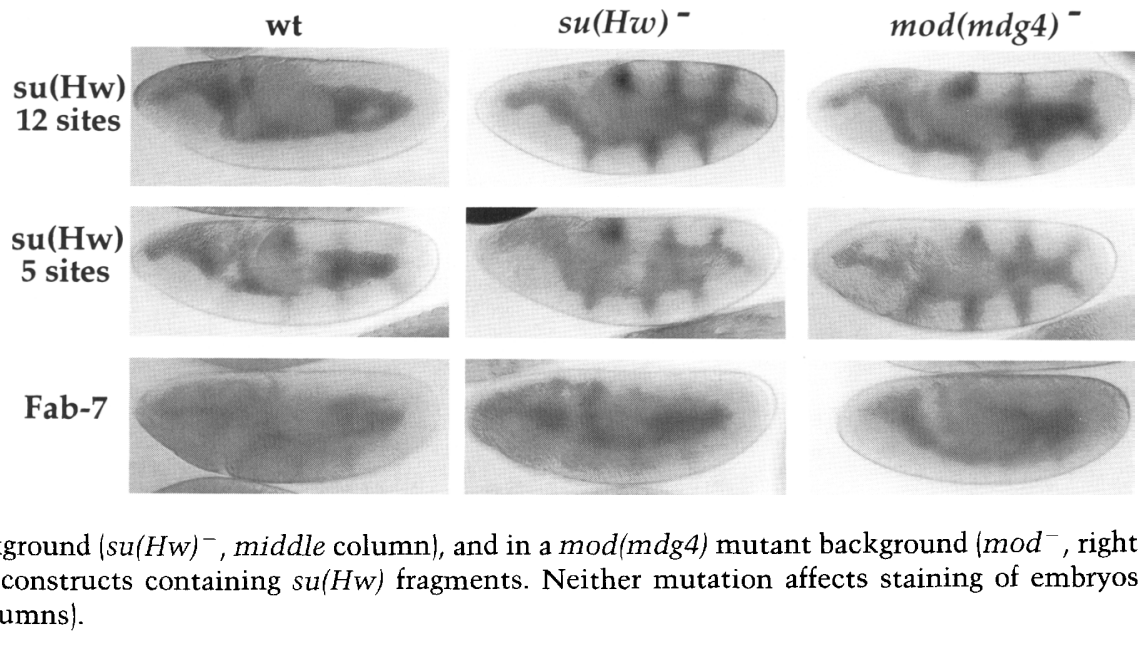


region of the bithorax complex is likely to contain such a boundary element (Gyurkovics et al. 1990; Galloni et al. 1993; Vazquez et al. 1993). This putative boundary appears to be required to separate the $i a b-6$ and $i a b-7$ cis-regulatory domains and small deletions in the Fab-7 region result in the fusion of these two domains into a single regulatory unit. This fused domain inappropriately regulates the $A b d-B$ homeotic gene in PS11, and typically causes a phenotypic transformation of PS11 into a copy of PS12.

In the studies reported here we have used enhancerblocking assays to determine whether the $\mathrm{Fab}-7$ region actually contains an element that can act as an autonomous chromatin domain boundary. We have found that a Fab-7 sequence spanning two major chromatin-specific nuclease-hypersensitive sites can function as a domain boundary in this assay, and block enhancer-promoter interactions. This same DNA sequence was implicated previously in the boundary activity of the Fab-7 region within the context of the bithorax complex /Galloni et al. 1993; Karch et al. 1994). The mapping of boundary function in the bithorax complex has been extended in more recent studies on the lac $Z$ expression pattern in mutants that delete Fab-7 boundary sequences but retain the Bluetail transposon (J. Mihaly, I. Hogga, H. Gyurkovics, J. Gausz, and F. Karch, in prep.). This in situ analysis defines a minimal element for boundary function in the bithorax complex that corresponds almost precisely to the sequences required for enhancer blocking in our transgene assays.

As has been observed for other insulators, enhancerblocking activity depends upon the position of the Fab-7 element relative to the enhancer and promoter. The Fab-7 element can prevent promoter activation only when interposed between the enhancer and the promoter, but has no effect on activation when the order is reversed and it is placed upstream of the enhancer and promoter. We have also found that the blocking activity of Fab-7 can not be attributed to some repressive or inhibitory effect of the element on either the promoter or the enhancer. Like other generic boundaries, blocking activity is not restricted to a specific enhancer or promoter, but can be detected in several different enhancerpromoter combinations. Similarly, the Fab-7 element is able to function as a boundary in a variety of cell and tissue types, and at different stages of development.

The results reported here demonstrate that Fab- 7 has many properties expected for an element that functions as a boundary of higher order chromatin domains. Whereas numerous other elements of this type have been identified, for the most part the biological functions of these putative domain boundaries have not yet been clearly established (Kellum and Schedl 1991; Chung et al. 1993; Roseman et al. 1993; for review, see Schedl and Grosveld 1995). In this respect, Fab-7 is an exceptional case as genetic studies have demonstrated that deletion of the Fab-7 boundary has profound consequences on the regulation of the homeotic $A b d-B$ gene. As such, Fab-7 would be one of the first examples of an element whose boundary activity is known to be essential in vivo.
The Fab-7 boundary is distinct from an adjacent PRE silencer

Two previous studies on transgenes containing a large DNA fragment from the Fab-7 region uncovered a potent negative regulatory activity (Busturia and Bienz 1993; Zink and Paro 1995). This finding led to the suggestion that Fab-7 is a gap and $P c-G$ gene dependent repressor rather than a boundary element (Busturia and Bienz 1993). Although we have also detected a $P c$ - $G$ dependent silencer in the $\mathrm{Fab}-7$ region, our experiments indicate that this silencer is completely distinct from the Fab-7 boundary. As shown in Figure 2A, we have found that the silencer is contained in a $0.8-\mathrm{kb}$ Aat I-Xba I restriction fragment located just distal to the site of insertion of the Bluetail transposon. In studies described in detail elsewhere (K. Hagstrom, M. Muller, and P. Schedl, in prep.) we have shown that this silencer corresponds to a PRE for the iab-7 cis-regulatory domain. Although we have not yet defined the precise limits of this $i a b-7$ PRE, it seem likely that nuclease hypersensitive site III (see Fig. 2A) is critical for silencing activity as it contains a conserved sequence found in PREs from other BX-C cisregulatory domains (Karch et al. 1994; unpubl.). Whereas the iab-7 PRE maps to the distal side of the Bluetail insertion site, we show here that sequences critical for boundary function in enhancer blocking assays are located to the proximal side of the transposon insertion site. The minimal Fab-7 boundary element defined by our assays includes two strong nuclease hypersensitive sites (HS1 and HS2), plus part of a minor hypersensitive site (Fig. 2).

Our functional dissection of the $\mathrm{Fab}-7$ region into a boundary element and a PRE provides a plausible explanation for why the phenotype of the original $F a b-7^{1}$ is slightly different from the two deletions, Fab- $7^{2}$ and $F a b$ $7^{3}$, generated by excision of the Bluetail transposon (Gyurkovics et al. 1990; Galloni et al. 1993) In Fab- $7^{1}$ the iab-7 cis-regulatory domain is ectopically activated in all PS11 cells and, in adults, this causes a complete transformation of abdominal segment A6 into A7. Although $i a b-7$ is also ectopically activated by the $F a b-7^{2}$ and $F a b$ $7^{3}$ mutations, this activation does not always occur and A6 has small clones of cells in which iab-7 remains off (Galloni et al. 1993; J. Mihaly, I. Hogga, H. Gyurkovics, J. Gausz, and F. Karch, in prep.).

As indicated in Figure $2 \mathrm{~A}$, the deletion in $\mathrm{Fab}-7^{1}$ removes not only the $F a b-7$ boundary but also the $i a b-7$ PRE. The loss of the boundary element results in the fusion of the $i a b-6$ and iab-7 cis-regulatory domains. Because the iab-7 PRE is also missing, the fused domain is primed to ectopically activate $i a b-7$ in PS11, leading to the complete transformation of PS11 into a copy of PS12 (A6 to A7). In contrast, the deletions in both Fab $-7^{2}$ and Fab $-7^{3}$ extend proximally from the Bluetail insertion site and remove the Fab-7 boundary, but leave the $i a b-7$ PRE intact. Like $F a b-7^{1}$, the removal of the boundary element results in a fusion of the $i a b-6$ and $i a b-7$ cisregulatory domains. However, because the $i a b-7$ PRE is still present in these mutants, the interactions between 
positive and negative initiation elements in the fused domain need not lead exclusively to the permanent activation of $i a b-7$ in PS11. Instead, there is a competition between ectopic activation of $i a b-7$ by positive elements in $i a b-6$ and ectopic silencing of $i a b-6$ by negative elements in iab-7 (J. Mihaly, I. Hogga, H. Gyurkovics, J. Gausz, and F. Karch, in prep.).

\section{Properties of the Fab-7 boundary}

In general, the properties of the Fab-7 element evident in the enhancer-blocking assays closely resemble those of classically defined insulators such as $s u(H w)$. In fact, the only major difference apparent in our experiments is that $\mathrm{Fab}-7$ does not require either the $s u(\mathrm{Hw})$ or the mod$(m d g 4)$ proteins. There are, however, some subtle differences that may ultimately be relevant to understanding how the Fab-7 boundary functions in the regulation of $\mathrm{BX}-\mathrm{C}$ gene expression. The most clear-cut is the relative strength of the blocking activity. This is best illustrated in the experiments with the $f t z: h s p 70 / l a c Z$ reporter where we compared the blocking activity of the Fab-7 element with $s u(H w)$ elements containing five and twelve binding sites. We found that the enhancer-blocking activity of the Fab-7 element is not as strong as the $s u(H w) 12$ mer but is stronger than the $s u(H w) 5$ mer. The intermediate strength of the Fab-7 boundary might also account for the position-dependent blocking in the white assay system (Vazquez and Schedl 1994). A second difference was noted in our analysis of the enhancerblocking activity of $\mathrm{Fab}-7$ in the CNS using the $\mathrm{ftz}$ : $\mathrm{ftz} /$ $1 a c Z$ reporter. Whereas the Fab-7 element reduced CNS $\beta$-galactosidase expression, it was clearly less effective in blocking the action of the $f t z$ NE enhancer in this reporter construct than it was in the ftz:hsp70/lacZ construct. By contrast, the enhancer-blocking activity of the $s u(H w) 12$ mer seemed to be relatively unaffected by the switch from the heterologous $h s p 70$ to the homologous $f t z$ promoter. This finding could indicate that the potency of the Fab-7 element depends upon the specific enhancer-promoter combination.

\section{Domain boundaries in the $B X-C$}

Although the Fab-7 boundary can block enhancer-promoter communication much like other insulators, this is not the normal function of Fab-7 in the bithorax complex. Rather, Fab-7 ensures the autonomous activity of the $i a b-6$ and $i a b-7$ cis-regulatory domains (Gyurkovics et al. 1990; Galloni et al. 1993). Early in development when parasegment identity is initially determined, the Fab-7 boundary must prevent adventitious interactions between gap and pair-rule gene products associated with each cis-regulatory domain. In the absence of the boundary, these adventitious interactions can lead to the inappropriate activation or inactivation of the cis-regulatory domains, and the improper regulation of $A b d-B$ expres- sion. As part of this process the Fab-7 boundary must also ensure that the maintenance system in each domain, the PRE (or TRE), is properly programmed. For example, in PS11, Fab-7 must prevent the gap and pairrule gene products associated with the iab-6 cis-regulatory domain from inappropriately inactivating the $i a b-7$ PRE. (A misprogrammed iab-7 PRE would not keep the iab-7 cis-regulatory domain silent in PS11, leading to the ectopic expression of $A b d-B$.) Once $\mathrm{BX}-\mathrm{C}$ regulation switches to the maintenance mode, the main function of the boundary is presumably to restrict PRE silencing for TRE activation) to the appropriate domain. Thus, in PS1 1 the $F a b-7$ boundary must prevent the $P_{C}-G$ proteins associated with the $i a b-7$ PRE in the silent iab-7 cisregulatory domain from ectopically silencing the adjacent $i a b-6$ domain.

An obvious expectation of this model is that the BX-C contains additional $\mathrm{Fab}$-7-like boundaries that block adventitious interactions between positive and/or negative regulatory elements in adjacent domains (see Fig. 1). In addition to their insulation function, these Fab-7-like boundaries could provide a mechanism for building functionally autonomous regulatory units with distinct parasegment specificity. The parasegmental specificity of each unit would be dictated by the particular combination of gap and pair-rule responsive elements that are included within the DNA segment delimited by a pair of Fab-7-like boundaries. Whereas the existence of other Fab-7-like boundary elements in BX-C has not been established, there are several potential candidates. These include the $M c p$ region that is located between $i a b-4$ and iab-5 (Karch et al. 1985) and an element (M. Galloni, S. Barges and F. Karch, pers. comm.) between iab-7 and $i a b-8$ that has enhancer-blocking activity in the miniwhite assay system (M. Muller, unpubl.).

Whereas Fab-7 ensures the independent activity of the $i a b-6$ and $i a b-7$ cis-regulatory domains, its ability to block enhancer-promoter communication when interposed between an enhancer and a promoter poses a problem. Why does this enhancer-blocking activity not prevent the more proximal $i a b-5$ and $i a b-6$ cis-regulatory domains from controlling the expression of the distal $A b d-B$ gene (see Fig. 1) in PS10 and PS11 (especially because $F a b-7$ can block these cis-regulatory domains from activating the Bluetail transposon)? One factor may be the strength of the Fab-7 boundary. The intermediate enhancer-blocking activity of the Fab-7 element might be sufficient to prevent cross-talk between the cis-regulatory domains, but insufficient to obstruct the proximal cis-regulatory domains from interacting with $A b d-B$. Another factor is suggested by the response of the Fab-7 and $s u(\mathrm{Hw})$ boundaries to different enhancer-promoter combinations. There may be specialized proteins that facilitate long-distance communication between these cis-regulatory domains and the $A b d-B$ promoter. Whereas a generic boundary like $s u(H w)$ might be able to interfere efficiently with these specialized proteins, the Fab-7 boundary may be designed so that it does not. Further studies will clearly be required to resolve this question. 


\section{Materials and methods}

\section{Cloning of P-element constructs}

white enhancer: mini-white The basic CaSpeR P-element construct is derived from the pRW vector described in Vazquez and Schedl (1994). It was modified to contain a minimal white enhancer fragment that drives expression in the eyc and testes, and extends only from BamHI to HindII (bp 4433-5540 in O'Hare ct al. 1984). Unique cloning sites were introduced upstream $(K p n I)$ and downstream (NotI and XhoI) of the enhancer. At the $3^{\prime}$ end of the mini-white gene is a 450-bp EcoRI to BamHI fragment of $\operatorname{scs}^{\prime}$ to minimize position effects (Kellum and Schedl 1992!. However, this scs' fragment is not a strong enhancer blocker and may not completely insulate the 3' end from position effects (Vazquez and Schedl 1994).

The Fab-7 fragments tested in constructs w\#3-12 were iso-

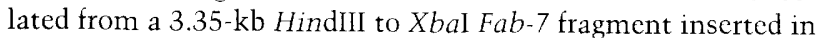
Bluescript. This Fab-7 region was originally isolated from phage $\lambda 8053$ (Karch et al. 1985) and spans bp 163-3517 as indicated in (Karch et al. 1994). The Fab-7 restriction fagments were cloned in a variety of ways; generally, if ends were not compatible they were modified with linkers to allow cloning into the unique sites.

The random DNA tested in $w \# 3$ is a $2.3-\mathrm{kb}$ Notl-Xhol fragment of Bic-D cDNA (from plasmid pc15a + described in Suter et al. 19891 .

ftz enhancers: hsp70 promoter/lacZ The basic CaSpeR P-element construct contains two enhancers from the $f t z$ regulatory region: the UPS and the NE. The enhancers were derived from within a $10-\mathrm{kb} K p n I$ fragment that contains the $f(z$ gene and its cis-regulatory regions (Hiromi et al. 1985). The upstream clement is a $2.7-\mathrm{kb} \mathrm{KpnI-Xbal} \mathrm{fragment,} \mathrm{and} \mathrm{the} \mathrm{neurogenic} \mathrm{en-}$ hancer is a Xbal-Ball fragment. The $h s p 70 / 1 a c Z$ reporter gene fusion is as described in Simon et al. (1985). The hsp70 promoter fragment begins at -194 (thus including regulatory elements such as the heat shock elements, the GAGA factor binding sites, and the basal promoter) and extends to include the first 37 amino acids of the hsp70 ORF (Simon et al. 1985). This fragment is fused to the lac $Z$ ORF. At the unique SacI sitc within lac $Z$ is a fusion to the remainder of $l a c Z$ and an $h s p 70$ trailer sequence (as found in plasmid pryFGH3 from Hiromi et al. 1985) A 450-bp EcoRI-BamHI fragment of $s c s^{\prime}$ is located just beyond the trailer sequences of the $h s p 70 /$ lac $Z$ reporter to minimize position effects (see above). The mini-white gene of CaSpeR is located at the $5^{\prime}$ end of the construct as a marker for transformation.

Various test fragments were cloned into this vector using unique NotI and XhoI sites between the enhancers and the promoter. The random DNA in fh\#1 is a $1.4-\mathrm{kb}$ Not $\mathrm{I}-$ HindIII fragment of Bic-D cDNA (from plasmid pc15a + described in Suter et al. 1989). The Fab-7 insert in fh\#2 is a $1.7-\mathrm{kb}$ ApaI-NcoI fragment (Fig. 2) that has enhancer blocking, but not silencing, function in the white enhancer:mini-white assay. The su(Hw) 12 fragment in fh\#4 is derived from a XmnI-Bsp 1286 fragment of the gypsy transposon |position 670-1041 in Spana et al. (1988) that includes 12 binding sites for the Su(Hw) protein]. The su(Hw) 5 fragment in fh\#3 includes only five $\mathrm{Su}(\mathrm{Hw})$ protein binding sites from within the same fragment (position 670830 in Spana et al. 1988) but in addition contains 195 bp of pUC18 sequences.

$\mathrm{ft} z$ enhancers:ftz promoter/lacZ This construct is identical to the $f t z: h s p 70 / 1 a c Z$ vector, except that the $h s p 70 / l a c Z$ reporter is replaced by the $f t z /$ lac $Z$ reporter. The $f t z$ promoter is originally derived from within a $10-\mathrm{kb} \mathrm{KpnI} \mathrm{ftz} \mathrm{gene} \mathrm{fragment}(\mathrm{Hi}-$ romi ct al. 1985). In addition to basal promoter elements, the $f t z$ promoter includes the zebra element, which in this construct extends to a HindIII site, 281 bp further $5^{\prime}$ than the zebra element described in Hiromi et al. (1985). This promoter fragment extends to amino acid position 270 of the $f t z$ ORF, includes four newly introduced amino acids, and is then fused to the fifth amino acid of $l a c Z$, and again ends with trailer sequences from $h s p 70$. The $F a b-7$ and $s u(H W) 12$ fragments tested in this vector are the same as those tested in the $f t z$ :hsp $70 / 1 a c Z$ vector.

\section{P-element transformation}

Of each P-element construct, $0.5 \mathrm{mg} / \mathrm{ml}$ was injected along with P-turbo helper plasmid (pUChspD2-3wc) into $w^{1}$ embryos, and transformants were selected by rescue of the $W^{-}$eye color. Crosses to marked balancer chromosomes were performed to generate balanced stocks and to determine the chromosome of insertion for each line.

\section{Scoring reporter gene expression}

mini-white To compare the relative levels of mini-white expression between different lines, homozygous transgenic lines were crossed back to $w^{1}$, and heterozygous progeny of the same sex and age were examined. (Heteroygous females that were 1 day old allowed the most sensitive detection of differences in cyc color.) The eye color of lines heterozygous for transgenes carrying the various fragments of Fab-7 were scored relative to the eye color of a control containing the basic construct (w\#2). All flies were raised at $22^{\circ}$.

$\beta$-galactosidase Embryos were collected at $0-15 \mathrm{hr}$ and stained overnight for $\beta$-galactosidase activity according to the protocol in (Bellen et al. 1989) except that embryos were fixed in one part PBS, one part 50\% gluteraldehyde, and two parts heptane. To facilitate comparison of relative levels, stainings were done simultaneously in the same dish, and the same positive and negative control lines were included each time staining was performed.

Heat shock of the $f t z: h s p 70 / 1 a c Z$ transgenic lines was performed by collecting $0-14 \mathrm{hr}$ embryos, transferring a portion of them to a prewarmed apple juice plate, incubating at $37^{\circ}$ for 1 hr, then staining as described.

\section{Testing su(Hw) and $\bmod (\operatorname{mdg} 4)$ mutant backgrounds}

The mutant strains used were: $y w f ; s u(H w)^{v}, b x^{34 e} / T M 6, U b x$, $\mathrm{su}(\mathrm{HW})^{f}$ (a null over a hypomorph), and $\bmod (\operatorname{mdg} 4)^{u 11} / \bmod -$ $(m d g 4)^{u 11}$ (nature of allele unknown) (Gerasimova et al. 1995). Males homozygous for th enhancer blocking constructs were crossed to $w^{T}$ females as a control or to mutant females, and $\beta$-galactosidase stainings of the resulting progeny were compared.

\section{Acknowledgments}

We thank T. Gerasimova and V. Corces for providing fly stocks and Yasushi Hiromi and Pam Geyer for providing DNA. We thank Julio Vazquez for providing the starting white enhancer: mini-white construct that was modified for use in our assays. We appreciate the assistance of Tala Villaruel de los Santos in cloning and Gretchen Calhoun in DNA sequencing. We thank all members of the lab for interesting and lively discussions. We wish to extend special thanks to members of the Karch lab and Gyurkovics and Gausz labs for the original observations that 
drove this project and their continued communication of ideas and unpublished results. This work was supported by National Institutes of Health grants (K.H. and P.S.) and a European Molecular Biology Organization long-term fellowship from the Schweizerische National Funds (M.M.).

The publication costs of this article were defrayed in part by payment of page charges. This article must therefore be hereby marked "advertisement" in accordance with 18 USC section 1734 solely to indicate this fact.

\section{References}

Bellen, H.J., C. O'Kane, C. Wilson, U. Grossniklaus, R.K. Pearson, and W.J. Gehring. 1989. P-element mediated enhancer detection: A versatile method to study development in Drosophila. Genes \& Dev. 3: 1288-1300.

Bienz, M. 1992. Molecular mechanisms of determination in Drosophila. Curr. Opin. Cell Biol. 4: 955-961.

Busturia, A. and M. Bienz. 1993. Silencers in Abdominal-B, a homeotic Drosophila gene. EMBO J. 12: 1415-1425.

Casares, F. and E. Sanchez Herrero. 1995. Regulation of the infraabdominal regions of the bithorax complex of Drosophila by gap genes. Development 121: 1855-1866.

Celniker, S.E., S. Sharma, D.J. Keelan, and E.B. Lewis. 1990. The molecular genetics of the bithorax complex of Drosophila: cis-regulation in the Abdominal-B domain. EMBO \%. 9: 4277-4286.

Chan, C.S., L. Rastelli, and V. Pirrotta. 1994. A Polycomb response element in the $U b x$ gene that determines an epigenetically inherited state of repression. EMBO I. 13:25532564.

Chung, I.H., M. Whiteley, and G. Felsenfeld. 1993. A 5' element of the chicken beta-globin domain serves as an insulator in human erythroid cells and protects against position effect in Drosophila. Cell 74: 505-514.

Duncan, I. 1987. The bithorax complex. Annu. Rev. Genet. 21: $285-319$.

Fauvarque, M.O. and J.M. Dura. 1993. polyhomeotic regulatory sequences induce developmental regulator-dependent variegation and targeted P-element insertions in Drosophila. Genes \& Dev. 7: 1508-1520.

Galloni, M., H. Gyurkovics, P. Schedl, and F. Karch. 1993. The bluetail transposon: Evidence for independent cis-regulatory domains and domain boundaries in the bithorax complex. EMBO /. 12: 1087-1097.

Gerasimova, T.I., D.A. Gdula, D.V. Gerasimov, O. Simonova, and V.G. Corces. 1995. A Drosophila protein that imparts directionality on a chromatin insulator is an enhancer of position-effect variegation. Cell 82: 587-597.

Gindhart, J.G., Jr. and T.C. Kaufman. 1995. Identification of Polycomb and trithorax group responsive elements in the regulatory region of the Drosophila homeotic gene Sex combs reduced. Genetics 139: 797-814.

Gyurkovics, H., J. Gausz, J. Kummer, and F. Karch. 1990. A new homeotic mutation in the Drosophila bithorax complex removes a boundary separating two domains of regulation. $E M B O$ I. 9: 2579-2585.

Hiromi, Y. and W.J. Gehring. 1987. Regulation and function of the Drosophila segmentation gene fushi tarazu. Cell 50: 963-974.

Hiromi, Y., A. Kuriowa, and W.J. Gehring. 1985. Control elements of the Drosophila segmentation gene fushi tarazu. Cell 43: 603-613.

Karch, F., B. Weiffenbach, M. Peifer, W. Bender, I. Duncan, S. Celniker, M. Crosby, and E.B. Lewis. 1985. The abdominal region of the bithorax complex. Cell 43: 81-96.

Karch, F., M. Galloni, L. Sipos, J. Gausz, H. Gyurkovics, and P. Schedl. 1994. Mcp and Fab-7: Molecular analysis of putative boundaries of cis-regulatory domains in the bithorax complex of Drosophila melanogaster. Nucleic Acids Res. 22: $3138-3146$.

Kassis, J.A., E.P. VanSickle, and S.M. Sensabaugh. 1991. A fragment of engrailed regulatory DNA can mediate transvection of the white gene in Drosophila. Genetics 128: 751-761.

Kellum, R. and P. Schedl. 1991. A position-effect assay for boundaries of higher order chromosomal domains. Cell 64: 941-950.

1992. A group of scs elements function as domain boundaries in an enhancer-blocking assay. Mol. Cell. Biol. 12: 2424-2431.

Kennison, J.A. 1993. Transcriptional activation of Drosophila homeotic genes from distant regulatory elements. Trends Genet. 9: 75-78.

Levis, R., T. Hazelrigg, and G.M. Rubin. 1985. Separable cisacting control elements for expression of the white gene of Drosophila melanogaster. EMBO I. 4: 3489-3499.

Lewis, E.B. 1978. A gene complex controlling segmentation in Drosophila. Nature 276: 565-570.

McCall, K., M.B. O'Connor, and W. Bender. 1994. Enhancer traps in the Drosophila bithorax complex mark parasegmental domains. Genetics 138: 387-399.

Modolell, J., W. Bender, and M. Meselson. 1983. D. melanogaster mutations suppressible by the suppressor of Hairy wing are insertions of a $7.3 \mathrm{~kb}$ mobile element. Proc. Natl. Acad. Sci. 80: 1678-1682.

Müller, J. and M. Bienz. 1992. Sharp anterior boundary of homeotic gene expression conferred by the fushi tarazu protein. EMBO /. 11: 3653-3661.

O'Hare, K., C. Murphy, R. Levis, and G. Rubin. 1984. DNA sequence of the white locus of Drosophila melanogaster. I. Mol. Biol. 180: 437-455.

Paro, R. 1990. Imprinting a determined state into the chromatin of Drosophila. Trends Genet. 6: 416-421.

Peifer, M. and W. Bender. 1988. Sequences of the gypsy transposon of Drosophila necessary for its effects on adjacent genes. Proc. Natl. Acad. Sci. 85: 9650-9654.

Peifer, M., F. Karch, and W. Bender. 1987. The bithorax complex: Control of segmental identity. Genes \& Dev. 1: 891898.

Pirrotta, V. 1988. Vectors for P-mediated transformation in Drosophila. In Vectors: A survey of molecular cloning vectors and their uses (ed. R.L. Rodriguez and D.T. Denhardt), pp. 437-456. Butterworth, Boston, MA.

Roseman, R., V. Pirrotta, and P. Geyer. 1993. The $s u(H w)$ protein insulates expression of the Drosophila melanogaster white gene from chromosomal position effects. $E M B O /$. 12: $435-442$.

Sanchez-Herrero, E. 1991. Control of the expression of the bithorax complex genes abdominal- $A$ and abdominal- $B$ by cis-regulatory regions in Drosophila embryos. Development 111: 437-449.

Schedl, P. and F. Grosveld. 1995. Boundaries and domains. In Chromatin structure and gene expression (ed. S.C.R. Elgin), pp. 172-196. IRL Press, New York, NY.

Scott, K. and P. Geyer. 1995. Effects of the su(Hw) insulator protein on the expression of the divergently transcribed Drosophila yolk protein genes. EMBO I. 14: 6258-6279.

Shimell, M.J., J. Simon, W. Bender, and M.B. O'Connor. 1994. Enhancer point mutation results in a homeotic transformation in Drosophila. Science 264: 968-971.

Simon, J. 1995. Locking in stable states of gene expression: 
Transcriptional control during Drosophila development. Curr. Opin. Cell Biol. 7: 376-385.

Simon, J.A., C.A. Sutton, R.B. Lobell, R.L. Glaser, and J.T. Lis. 1985. Determinants of heat shock induced chromosomal puffing. Cell 40: 805-817.

Simon, J., M. Peifer, W. Bender, and M. O'Connor. 1990. Regulatory elements of the bithorax complex that control expression along the anterior-posterior axis. EMBO /. 9:39453956.

Simon, J., A. Chiang, W. Bender, M.J. Shimell, and M. O'Connor. 1993. Elements of the Drosophila bithorax complex that mediate repression by Polycomb group products. Dev. Biol. 158: 131-144.

Smith, P.A. and V.G. Corces. 1992. The $s u(H w)$ binding region is required for gypsy mutagenesis. Mol. Gen. Genet. 233: 6570.

Spana, C., D.A. Harrison, and V.G. Corces. 1988. The Drosophila melanogaster suppressor of Hairy-wing protein binds to specific sequences of the gypsy retrotransposon. Genes \& Dev. 2: 1414-1423.

Suter, B., L. Romberg, and R. Steward. 1989. Bicaudal-D, a Drosophila gene involved in developmental asymmetry: Localized transcript accumulation in ovaries and sequence similarity to myosin heavy chain tail domains. Genes \& Dev. 3: $1957-1968$.

Vazquez, J. and P. Schedl. 1994. Sequences required for enhancer blocking activity of scs are located within two nucleasehypersensitive regions. EMBO I. 13: 5984-5993.

Vazquez, J., G. Farkas, M. Gaszner, A. Udvardy, M. Muller, K. Hagstrom, H. Gyurkovics, L. Sipos, J. Gausz, M. Galloni, et al. 1993. Genetic and molecular analysis of chromatin domains. Cold Spring Harbor Symp. Quant. Biol. 58: 45-54.

Zink, D. and R. Paro. 1995. Drosophila Polycomb-group regulated chromatin inhibits the accessibility of a trans-activator to its target DNA. EMBO J. 14: 5660-5671. 


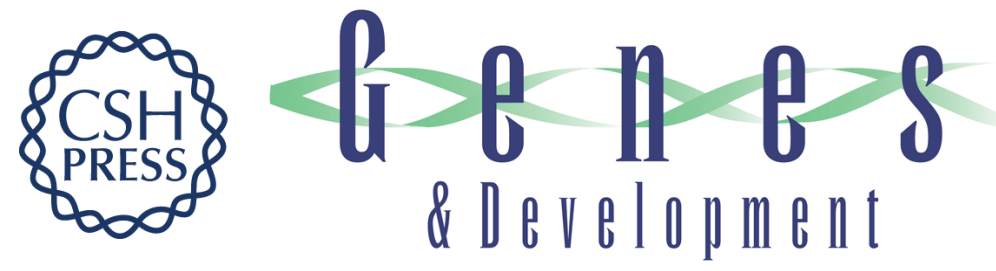

\section{Fab-7 functions as a chromatin domain boundary to ensure proper segment specification by the Drosophila bithorax complex.}

K Hagstrom, M Muller and P Schedl

Genes Dev. 1996, 10:

Access the most recent version at doi:10.1101/gad.10.24.3202

References This article cites 44 articles, 15 of which can be accessed free at:

http://genesdev.cshlp.org/content/10/24/3202.full.html\#ref-list-1

License

Email Alerting

Service

Receive free email alerts when new articles cite this article - sign up in the box at the top right corner of the article or click here.

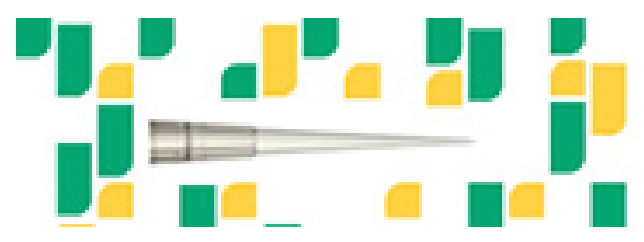

Focused on your science. 